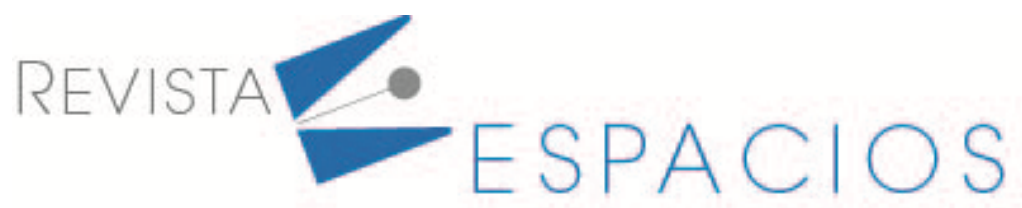

\title{
Uso de acuerdos comerciales: Caso Nuevo León (México)
}

\author{
Use of trade agreements: Case Nuevo León (Mexico)
}

\author{
LECHUGA-CARDOZO, Jorge Isaac ${ }^{1}$ \\ LEYVA-CORDERO, Oswaldo ${ }^{2}$ \\ PAMANES-DURAN, Alejandro Raisuli ${ }^{3}$
}

\begin{abstract}
Resumen
El objetivo de este artículo es mostrar evidencia del aprovechamiento de los acuerdos comerciales en Nuevo León (México). Se definió un estudio cualitativo de tipo analítico, no experimental, transeccional y de campo. Se diseñó un instrumento, y se aplicó a una muestra de nueve expertos en Negocios Internacionales. Se concluye que la firma de acuerdos comerciales en México abre un abanico de oportunidades como preferencias arancelarias, ampliación de mercado, y abaratamiento en materias primas.
\end{abstract}

Palabras clave: Acuerdos comerciales, diversificación de exportaciones, mecanismos de promoción comercial.

\begin{abstract}
The objective of this article is to show evidence of the use of commercial agreements in Nuevo León (Mexico). A qualitative study of analytical, non-experimental, transectional and field type was defined. An instrument was designed and applied to a sample of nine experts in International Business. This questionnaire presents five, three and two items for the variables export diversification, trade agreements and trade promotion mechanisms respectively. It is concluded that the signing of trade agreements in Mexico, opens a range of opportunities such as tariff preferences, market expansion, and cheaper raw materials.
\end{abstract}

key words: Export diversification, Trade agreements, trade promotion mechanisms.

\section{Introducción}

En México, el aprovechamiento de los acuerdos comerciales representa un importante aporte al crecimiento económico, la balanza de pagos, el empleo y la redistribución del ingreso (Vinesh et al, 2014, p.12).

Con la apertura económica y, por ende, la internacionalización de la economía mexicana entre la década de los ochenta y noventa se establece un cambio de modelo económico, pasando de un modelo proteccionista a uno de libre mercado. En este sentido, en 1986 México ingresa formar parte del Acuerdo General sobre Aranceles Aduaneros y Comercio o GATT. Pero es hasta el aumento más importante en el grado de apertura cuando se dio con la firma del Tratado de Libre Comercio para América del Norte (TLCAN) en 1994 (Schiavon y Ortiz, 2001),

\footnotetext{
${ }^{1}$ Doctorando en Relaciones internacionales, Negocios y Diplomacia. Facultad de Ciencias Políticas y Relaciones Internacionales. Universidad Autónoma de Nuevo León, México. Email: jorge.lechugacrd@uanl.edu.mx

${ }^{2}$ Subdirector de Posgrados. Facultad de Ciencias Políticas y Relaciones Internacionales. Universidad Autónoma de Nuevo León, México

${ }^{3}$ Doctorando en Relaciones internacionales, Negocios y Diplomacia. Facultad de Ciencias Políticas y Relaciones Internacionales. Universidad Autónoma de Nuevo León, México. Email: apamanesda@uanl.edu.mx
} 
alcanzando niveles de apertura superiores al 50\% (Gómez, 2013). En contraste Villareal y Ramos (2001) afirman que México se enfrenta a la paradoja de la competitividad: es uno de los países más abiertos al mantener acuerdos de libre comercio con países de tres continentes y, pero es de los menos competitivos.

Como bien apunta Martínez (2007), aunque México participa del comercio internacional desde los años ochenta, lo han realizado a través de productos tradicionales como materias primas, poco industrializados, a partir de la apertura económica se importa gran cantidad de tecnología, pero, hasta el día de hoy, las exportaciones no se mueven a la producción de bienes de mayor complejidad y sofisticación que permitan el ingreso a mercados con mayores exigencias de innovación.

En el marco de este proceso de integración económica mundial (Lechuga, 2016, p. 5) México ha firmado diferentes acuerdos comerciales con el objetivo de entrar de lleno al comercio internacional, actualmente cuenta con trece acuerdos de libre comercio que dan acceso a diferentes economías y, por consiguiente, a millones de habitantes o consumidores. Sin embargo, estos están siendo descapitalizados si tenemos en cuenta que, de acuerdo con el Observatorio de Complejidad Económica, la participación en exportaciones superiores al $73 \%$ con Estados Unidos y 5,2\% con Canadá proviene de un acuerdo principalmente TLCAN recientemente renombrado USMCA (Ver Tabla 1).

Tabla 1

Destinos de Exportación de México al mundo

\begin{tabular}{lc}
\hline \multicolumn{1}{c}{ Destino de Exportación } & Participación \\
\hline Estados Unidos & $73 \%$ \\
Canadá & $5.2 \%$ \\
Alemania & $2.1 \%$ \\
China & $2.1 \%$ \\
Japón & $1.3 \%$ \\
España & $1.1 \%$ \\
Otros & $15,2 \%$ \\
Total & $100 \%$ \\
\hline
\end{tabular}

Fuente: Elaboración propia (2019).

Nota: Datos Observatorio de Complejidad Económica MIT, (2017).

En el presente artículo se revisa el caso de cómo en Nuevo León (México) se están aprovechando los acuerdos comerciales, la diversificación de exportaciones que incluyen los factores identificados tales como económicos, humanos y sociales, así como los mecanismos de promoción comercial. Dichos aportes teóricos facilitan la explicación del fenómeno de la exportación (Álvarez y Myro, 2018) en el estado de Nuevo León.

En concreto, el desarrollo exportador y la mejoría de la capacidad empresarial que le acompaña, cambiaron en tres décadas el paisaje de la plataforma productiva en Nuevo León. En Nuevo León la conformación de clúster más relevantes se ha hecho en torno a un núcleo exportador de manufacturas industriales, que han ido avanzando con mayor o menor profundidad en la cadena de valor (Guardia, 2009).

Con relación a los sectores que están aprovechando los acuerdos comerciales de acuerdo con Data Nuevo León de la Secretaría de Economía y Trabajo (2018), son en su orden: Equipo de transporte, equipo de generación eléctrica, electrodomésticos, Computación, comunicación y navegación, productos metálicos, maquinaria y equipo, alimentos, bebidas y tabaco, productos químicos, metálicas básicas, y minerales no metálicos (Secretaría de Economía y Trabajo-Nuevo León, 2018).

En este estudio se identifica como problema una debilidad en el comercio internacional generada por la falta de aprovechamiento de los acuerdos comerciales firmados por México Observatorio de Complejidad Económica y autores como (López, 2015; Vinesh et al, 2014; Agosin, 2009; Acosta et al, 2003 y Casas, 2015). En este sentido, 
se plantea como pregunta problema: ¿Cuál es el estado actual de los acuerdos comerciales, la diversificación de exportaciones y los mecanismos de promoción comercial en el Estado Nuevo León (México)?

El objetivo del artículo es mostrar evidencia del aprovechamiento de los acuerdos comerciales en Nuevo León (México). Se realiza un estudio uni-variado en el cual se identifican como variables los acuerdos comerciales, la diversificación de exportaciones y los mecanismos de promoción comercial. Esta es una investigación de enfoque cualitativo utilizando la técnica de la entrevista a expertos, corresponde a un diseño no experimental, de corte trasversal o seccional y alcanza un nivel analítico en el espacio geográfico de Nuevo León. A continuación, se definen las variables acuerdos comerciales, diversificación de exportaciones y mecanismos de promoción comercial a partir de la revisión de los principales autores en el tema.

\subsection{Los Acuerdos Comerciales en México}

Córdoba (2015) define los acuerdos comerciales como el acuerdo mediante el cual dos o más países reglamentan de manera comprehensiva sus relaciones comerciales, con el objeto de aumentar los flujos de comercio e inversión, así como desarrollo económico y social (p.31).

Es decir, la eliminación o rebaja sustancial de los aranceles para los bienes y el establecimiento de acuerdos para la prestación de servicios entre las partes. Bajo las reglas de la Organización Mundial del Comercio o por mutuo acuerdo entre los países, como se muestra en el cuadro 1.

Cuadro 1

Acuerdos Comerciales de México

\begin{tabular}{lc}
\hline \multicolumn{1}{c}{ Acuerdo Comercial } & Año \\
\hline TLCAN & 1994 \\
Colombia & 1995 \\
Chile & 1999 \\
Israel & 2000 \\
Unión Europea & 2000 \\
Islandia, Liechtenstein, Noruega y Suiza & 2001 \\
Uruguay & 2004 \\
Japón & 2005 \\
Perú & 2012 \\
Centroamérica & 2013 \\
Panamá & 2015 \\
Alianza del Pacifico & 2016 \\
TPP & En proceso \\
\hline \multicolumn{2}{c}{ Fuente: Secretaría de Economía, (2015). }
\end{tabular}

Por una parte, Lombana et al (2013) para que un acuerdo sea reconocido debe tener un cubrimiento sustancial del comercio entre los miembros. Así como los países mantienen su política exterior fuera de los límites del acuerdo, de modo que los aranceles intra-acuerdo, pero con terceros países (fuera del acuerdo) pueden ser distintas.

Agrega Guardia (2009) los Tratados de Libre Comercio (TLC) trascienden el ámbito estrictamente comercial (barreras arancelarias y no arancelarias, normas de origen), en la medida que incorporan otras disciplinas comerciales tales como: servicios, inversión, propiedad intelectual, compras gubernamentales, medio ambiente, normas laborales, cooperación, y mecanismos de resolución de controversias (Guardia, 2009).

De este modo Córdoba (2015) resalta que estos sirven para promover un entorno estable y sin barreras para el comercio y la inversión, garantizado el acceso de productos y servicios del país a mercados externos. Al ser aprovechado por los empresarios nacionales, permite que la economía del país crezca, aumente la 
comercialización de productos nacionales, se genere más empleo, se modernice el aparato productivo, mejore el bienestar de la población y se promueva la creación de nuevas empresas por partes de inversionistas extranjeros (p.35).

Ahora bien, algunas de las bondades derivadas de la implementación de acuerdos comerciales derivan de las preferencias arancelarias en doble vía, lo que se percibe como una ampliación de mercado para las partes y aprovechamiento de escalas de producción para las empresas, se generan eslabonamientos productivos antes impensables, para su aprovechamiento es necesario innovar y adaptar la institucionalidad económica (Giraldo, 2006; Trujillo, 2014).

Igualmente se espera que los acuerdos estén seguidos por aumento en las exportaciones e importaciones más baratas provenientes de los países con los que suscriben dichos acuerdos, el riesgo de desviación se disminuye cuando existe una red de acuerdos sobre todo con países desarrollados, lo que repercute en beneficios para el consumidor y una disminución en costos para las empresas que aprovechan insumos de los socios comerciales. Los primeros beneficiados son las grandes empresas y productos que están presentes en los mercados internacionales y que con las nuevas preferencias tienen posibilidad de ampliar su participación en el mercado (Solorza, 2012).

Al mismo tiempo de acuerdo con la teoría económica un país que liberaliza su comercio debería teóricamente aumentar su ritmo de crecimiento, desarrollar salarios más elevados, flujos más grandes de capital externo, ganancias de productividad y transferencia tecnológica (Guardia, 2009, p. 70).

\subsection{Aproximación a la diversificación de exportaciones en el estado de Nuevo León}

En primer lugar, Zapata et al (2011) define a la estrategia de exportación como la forma más corriente de comercio internacional de productos básicos, materias primas, productos alimenticios y de algunos servicios y productos manufacturados o como lo plantea Lamb et al (2006) es la "venta de bienes de producción nacional a compradores que están en otro país" (p.15).

Por otro lado, Aulakh, Kotabe y Teengen (2000), definen la estrategia exportadora partir de tres dimensiones: "las estrategias competitivas, el grado de adaptación o estandarización de las actividades de marketing en los mercados exteriores y la estrategia de expansión, esto es, el grado de diversificación o alcance geográfico de las exportaciones" (p.43).

En contraste Thompson et al (2004), asocian el éxito de la estrategia a largo plazo a la competitividad relativa en costos de la base de producción en el país de origen. Una estrategia de exportación tiene éxito mientras las ventajas de exportación sean mayores que sus desventajas (p.211).

En este sentido expresa Martín (2004), la elección de la estrategia de exportación depende tanto de factores internos de la empresa, como el tamaño, la experiencia internacional o la pertenencia de la empresa a grupo empresarial; como de factores del sector industrial, como el grado de concentración o la existencia de clientes internacionales en la industria; y de factores relacionados con el país de destino, como el tamaño del mercado o el riesgo (p.33).

Así la estrategia de expansión en la que se centra nuestro estudio es definida por Fernández et al (2008) como al "número de mercados exteriores a los que se dirige una empresa exportadora y que constituye una decisión estratégica que puede tener implicaciones importantes para el resultado global de su actividad exportadora" (p.43).

De modo que Aulakh, Kotabe y Teengen (2000) asegura que el número de mercados que una empresa exportadora atiende es una decisión estratégica que puede tener implicaciones importantes para el éxito 
exportador en su conjunto. Asimismo, Piercy (1982) afirma que la selección del número de mercados en los que estar presente reviste una gran importancia ya que, dependiendo de la amplitud de países que se desea atender, supondrá la concentración o dispersión de los esfuerzos a realizar por la empresa (Flor et al, 2010, p.17).

Agrega Flor et al (2010) las compañías apropian una estrategia de expansión de mercados si optan por tener presencia en una amplia cantidad de países. Esta estrategia posee ventajas relacionadas a menor dependencia en relación a la concentración en un solo mercado. Estudios de Cooper y Kleinschmidt (1985) entre otros concuerdan que existe una relación positiva entre la diversificación de mercado y el desempeño exportador de la empresa. No obstante, otras investigaciones como la de Reid presentan resultados favorables a favor de la concentración de mercado, así como Madsen asegura que las empresas deben explotar los mercados que ya atienden, en vez de dispersar sus esfuerzos en un amplio número de países (Flor et al, 2010, p.17).

Así pues, son varios los elementos que pueden llevar a una empresa a internacionalizarse, como lo asegura Flor et al (2010) "Comportamiento de la empresa exportadora ha captado el interés de numerosas investigaciones, buena parte de las cuales se han destinado a identificar la influencia que diferentes factores ejercen sobre el resultado de su actividad exterior, al que denomina desempeño exportador" (p. 15).

\subsection{Factores asociados a la diversificación en Nuevo León}

Escandón et al (2014) centra su estudio en 6 grupos de factores determinantes de la internacionalización temprana de pequeñas empresas o fenómeno Born Global: "características del emprendedor (fundador), factores internos de la empresa, características del sector, características del entorno regional, recursos y capacidades e innovación" (Escandón et al, 2014, p.175).

Así, Fernández et al (2008) plantea que en la literatura existen diferentes factores/variables, que influyen en el resultado de la exportación, el gran número modos en que dicho factores son medidos y la carencia de un marco teórico consistente que guíe la selección de dichos factores resulta un problema (p.41).

A partir de lo anterior, en el presente estudio se han agrupado dichos factores en tres grandes grupos a saber; factores humanos, factores económicos y factores sociales. A continuación, se realiza una aproximación teórica de cada uno.

Factores Financieros. En cuanto a la insuficiencia de fondos financieros puede ocasionar que las empresas tengan menos expectativas para vender en el mercado internacional, ausencia oportunidades para invertir en instalaciones productivas, innovación y desarrollo, o desarrollar economías de escala. Lo que restringe o limita una participación exitosa en los mercados internacionales (Cabrear y Olivares, 2012; Benito y Welch, 1994; Deardoff, 1984; Moen, 1999; Zahra et al., 2003).

Factores Humanos. En efecto, algunos autores aseguran que la formación, la educación y la práctica de los empleados determinan las capacidades disponibles para las empresas (Grant, 2002). En este sentido, diversos estudios indican una relación positiva entre las capacidades de los principales decisores en las empresas y el resultado exportador (Cabrear y Olivares, 2012; Davis y Harveston, 2000; Reuber y Fischer, 1997; Westhead et al., 2001).

Factores Sociales. Por otra parte, la asociatividad puede incrementar la penetración internacional de la empresa porque a través de esta es posible determinar áreas de oportunidad para introducir cambios en los productos de la empresa, reducir la incertidumbre e incrementar el conocimiento de la empresa en relación a su entorno (Westhead et al., 2001, 2004; Zahra, Matherne y Carleton, 2003). Adicionalmente las relaciones internacionales de colaboración y los vínculos están relacionados directamente con el crecimiento internacional de la empresa. 


\subsection{Aproximación a los Mecanismos de Promoción Comercial en el Estado de Nuevo León}

Por otro lado, se encuentran la promoción de exportaciones, definida por Seringhaus (1986) como las medidas de política pública que actual o potencialmente conducen a la actividad exportadora al nivel empresarial, industrial o nacional. Aunque ésta puede ser proporcionada por organismos públicos, privados o mixtos (p.55), Wheeler (1990), argumenta que muchas empresas son incapaces de realizar las exportaciones agresivamente por la carencia de experiencia, por sus recursos limitados o por otro obstáculo percibido: barreras para la exportación. Por lo tanto, los programas de promoción de exportaciones son suministrados por el gobierno, asociaciones comerciales $u$ otras organizaciones, para ayudar a las empresas a superar dichas barreras, especialmente, a las Pymes (Martínez, 2007, p. 7).

Agregan Lombana et al (2013) ProMéxico fomenta promociona las exportaciones mexicanas, facilita la transferencia tecnológica, el desarrollo del capital humano y la inversión en I+D con el propósito de promover el establecimiento de empresas principalmente medianas fuera del país a través de asesorías especializadas, asistencia a viajes de negocio, capacitación de talento humano y practicantes en el exterior (p. 189).

\section{Metodología}

\subsection{Diseño}

La investigación en la que se origina este artículo se realizó desde un enfoque cualitativo, con una metodología cualitativa de tipo analítica y un diseño no experimental, transeccional y de campo. Las variables trabajadas (diversificación de exportaciones, acuerdos comerciales y mecanismos de promoción comercial) se analizaron a través de expertos en negocios internacionales en la ciudad de Monterrey (México).

El enfoque de la presente investigación es cualitativo, ya que las técnicas que se utilizarán para la recolección de datos como la entrevista, ayudarán a recopilar información y como bien lo dicen Taylor y Bogdán (1986) "es aquella que produce datos descriptivos: las propias palabras de las personas, habladas o escritas, y la conducta observable".

En este sentido Autores como Mendoza (2006) destacan la importancia de la investigación cualitativa en la medida que permite descubrir y refinar las preguntas de investigación que guiarán a los instrumentos utilizados para la obtención de la información. Así como Rodríguez y Gorjón (2014) resaltan que da un sentido profundo de conocimiento acerca de las actitudes, comportamiento, percepciones y preferencias de las personas (p. 169).

El alcance establecido es el analítico o interpretativo, pretende encontrar pautas de relación internas en un evento para llegar a un conocimiento más profundo de-éste, que la mera descripción. Para ello se vale de las matrices de análisis, que proporcionan los criterios que permiten identificar esas pautas de relación. La investigación analítica intenta desentrañar lo que está más allá de lo evidente (Hurtado, 2010, p. 133). Agrega Hernández, Fernández y Baptista (2010) la investigación interpretativa intenta encontrar sentido a los fenómenos en función de los significados que las personas les otorguen (p. 9).

El diseño de investigación escogido es el transversal, porque el estudio que se busca hacer no tendrá un seguimiento en el tiempo y se efectuará en un periodo determinado (Hernández, Fernández y Baptista, 2014, p. 154).

\subsection{Participantes}

La población de referencia considerada son los expertos en negocios internacionales en Monterrey. Para el presente trabajo se seleccionaron 9 expertos en negocios internacionales en Monterrey seleccionados de acuerdo con la disposición de atención al libreto de entrevista en el campo. La selección de expertos se realiza 
con base a las siguientes características: Disponibilidad y accesibilidad para contestar la entrevista, conocimiento técnico del fenómeno de la exportación y los acuerdos comerciales en México, ubicación geográfica en Nuevo León, y formación académica mínima de doctor o su equivalente en experiencia en campo.

Cabe resaltar que la utilización de $n$ cantidad de expertos es válida en la medida que una muestra cualitativa busca significados (Martínez-Salgado, 2012), que permitan lo que Sandelowski y Barroso (2003) Ilaman generalización ideográfica. El criterio más generalmente compartido en metodología cualitativa es el de la saturación, que tiene lo que Carlsen y Glenton (2011) llaman un rol dominante, aunque difícil de operativizar. La propuesta consiste en que dejamos de recoger unidades muestrales en el trabajo de campo cuando las nuevas unidades seleccionadas dejan de aportar nueva información relevante con respecto a las anteriores (GarcíaHernández y Manzano-Caudillo, 2010).

\section{Cuadro 2}

Expertos a los que se

les aplicó la entrevista

\begin{tabular}{cll}
\hline Informador & \multicolumn{1}{c}{ Puesto } & \multicolumn{1}{c}{ Institución } \\
\hline 1 & Director General & $\begin{array}{l}\text { Consejo Mexicano de Comercio Exterior (COMCE } \\
\text { Noreste) } \\
\text { Embajada de Tailandia en México }\end{array}$ \\
2 & $\begin{array}{l}\text { Primera secretaria (Asuntos de Protocolo, } \\
\text { Consulares y Económicos) } \\
\text { Consejero Comercial de Filipinas (Oficina Comercial, } \\
\text { Centro de Comercio e Inversión Filipinas) } \\
\text { Investigador en Competitividad de los Negocios } \\
\text { Internacionales, Perfil Proded }\end{array}$ & $\begin{array}{l}\text { Embajada de Filipinas } \\
\text { en México } \\
\text { Universidad Autónoma de Nuevo León }\end{array}$ \\
5 & $\begin{array}{l}\text { Investigador en Competitividad de los Negocios } \\
\text { Internacionales, Perfil Proded }\end{array}$ & Universidad Autónoma de Nuevo León \\
6 & $\begin{array}{l}\text { Investigador Líder del Cuerpo Académico Gestión de } \\
\text { la Innovación en Modelos de Negocios } \\
\text { Investigador en Competitividad de los Negocios }\end{array}$ & Universidad Autónoma de Nuevo León \\
8 & $\begin{array}{l}\text { Internacionales, Perfil Proded -SNI I } \\
\text { Investigador en Economía Regional y Urbana - }\end{array}$ & Universidad Autónoma de Nuevo León \\
9 & $\begin{array}{l}\text { Director de la Facultad de Economía } \\
\text { Agente Aduanal - Propietario }\end{array}$ & Universidad Autónoma de Nuevo León \\
\hline & \multicolumn{2}{c}{ Fuente: Elaboración propia, (2019). }
\end{tabular}

Por otro lado, agregan Creswell y Miller (2000) que en la investigación cualitativa los resultados son precisos desde el punto de vista del investigador, el participante o los lectores de un informe de investigación, así como en palabras de Strauss y Corbín (1990) sostiene que en la teoría fundamentada se da la validez suplementaria al relacionar datos con categorías y contrastar los datos con la literatura. De modo que la validez interna está dada por los autores y la validez externa por la calidad del experto. 


\section{Cuadro 3}

Ficha técnica de

la investigación

Universo

Ámbito

Método de recogida de información

Unidad muestral

Tamaño de la muestra

Trabajo de campo

Número de entrevistas realizadas

Respuestas válidas
Expertos en Negocios Internacionales en Monterrey (México) Regional, Monterrey (México)

Entrevista semi- estructurada, suministrada presencialmente Expertos en Negocios Internacionales en Monterrey (México)

9

Octubre de 2019

9

9

Fuente: Elaboración propia, (2019).

\subsection{Instrumentos}

Se diseñó un instrumento en conformidad con las tres variables (diversificación de exportaciones, acuerdos comerciales y mecanismos de promoción comercial), el cual se aplicó a 9 expertos en negocios internacionales en Monterrey. Dicho instrumento ofrece 10 ítems con respuestas abiertas. El instrumento se construyó con base en las dimensiones evaluadas en la investigación.

De modo que la técnica utilizada, la entrevista, está orientada a establecer contacto directo a con las personas que se consideren fuente de información. Si bien puede soportarse en un cuestionario flexible, su propósito es obtener información espontánea y abierta (Bernal, 2010, p. 177).

\section{Cuadro 4}

Estructura del guión

de la entrevista

\begin{tabular}{lcl}
\hline \multicolumn{1}{c}{ Categorías de Análisis } & $\begin{array}{c}\text { Número de } \\
\text { preguntas }\end{array}$ & \multicolumn{1}{c}{ Autores } \\
\hline Diversificación de exportaciones & 5 & $\begin{array}{l}\text { Zapata et al (2011), Fernández et al (2008), } \\
\text { Thompson et al (2004), López-Cózar (2013), Flor } \\
\text { et al (2010), Escandón et al (2014) } \\
\text { Córdoba (2015), Lombana et al (2013), Guardia } \\
\text { (2007) } \\
\text { Mecuerdos comerciales }\end{array}$ \\
& 3 & $\begin{array}{l}\text { Martínez (2007) } \\
\text { Centro de Análisis Estratégico para la Agricultura } \\
\text { (s.f.) }\end{array}$ \\
\hline
\end{tabular}

Fuente: Elaboración propia, (2019). 


\section{Cuadro 5}

Cuestionario e indicadores

de la investigación cualitativa

\begin{tabular}{|c|c|c|}
\hline - Número & - Pregunta & - Dimensión \\
\hline-1 & $\begin{array}{l}\text { ¿Cuál es la importancia de conocer los acuerdos } \\
\text { comerciales? }\end{array}$ & $\begin{array}{l}\text { Diagnóstico de la diversificación (acceso a } \\
\text { mercados) }\end{array}$ \\
\hline-2 & $\begin{array}{l}\text { ¿Qué es más importante mayor o menor } \\
\text { diversificación de exportaciones? }\end{array}$ & $\begin{array}{l}\text { Diagnóstico de la diversificación (alcance } \\
\text { geográfico) }\end{array}$ \\
\hline-3 & $\begin{array}{l}\text { ¿Están las empresas exportadoras de su región } \\
\text { aprovechando los acuerdos comerciales? ¿Por qué? }\end{array}$ & $\begin{array}{l}\text { Mecanismos de promoción comercial para } \\
\text { la diversificación (acceso a mercados) }\end{array}$ \\
\hline-4 & $\begin{array}{l}\text { ¿Cuáles son los obstáculos para que las empresas de } \\
\text { su región no se beneficien de los acuerdos } \\
\text { comerciales? }\end{array}$ & $\begin{array}{l}\text { Mecanismos de promoción comercial para } \\
\text { la diversificación (competitividad) }\end{array}$ \\
\hline-5 & $\begin{array}{l}\text { ¿Cuál es la política comercial internacional de su } \\
\text { país? }\end{array}$ & $\begin{array}{l}\text { Mecanismos de promoción comercial para } \\
\text { la diversificación } \\
\text { (gobernabilidad) }\end{array}$ \\
\hline-6 & $\begin{array}{l}\text { ¿Cuáles son los mecanismos de Promoción } \\
\text { Comercial en su región? }\end{array}$ & $\begin{array}{l}\text { Mecanismos de promoción comercial para } \\
\text { la diversificación (gobernabilidad) }\end{array}$ \\
\hline-7 & $\begin{array}{l}\text { ¿De acuerdo a su experiencia cuáles son los factores } \\
\text { que inciden en la diversificación de exportaciones en } \\
\text { su región? }\end{array}$ & $\begin{array}{l}\text { Identificación de los factores de } \\
\text { diversificación (factores financieros, } \\
\text { humanos y sociales) }\end{array}$ \\
\hline-8 & ¿Cómo evaluaría la diversificación en su región? & $\begin{array}{l}\text { Evaluación de diversificación en empresas } \\
\text { (diversificación) }\end{array}$ \\
\hline-9 & $\begin{array}{l}\text { ¿Qué indicadores utilizaría para medir la } \\
\text { diversificación en su región? }\end{array}$ & $\begin{array}{l}\text { Evaluación de diversificación en empresas } \\
\text { (diversificación) }\end{array}$ \\
\hline-10 & $\begin{array}{l}\text { ¿Qué similitudes y diferencias encuentra en el } \\
\text { comportamiento de la diversificación de } \\
\text { exportaciones de su región con otras regiones de } \\
\text { América Latina? }\end{array}$ & $\begin{array}{l}\text { Comportamiento de factores de } \\
\text { diversificación (diversificación) }\end{array}$ \\
\hline
\end{tabular}

Fuente: Elaboración propia, (2019).

\subsection{Procedimiento}

Para entender el problema se realiza una recopilación de fuentes secundarias, una vez compilada la información se construye el artículo. luego se levanta la información primaria a través del uso de la técnica la entrevista a expertos y el instrumento guion de entrevista semiestructurado que sirve para la recolección de dicha información. De modo que recolectada la información primaria se tabuló la información a través de software Nvivo y se expresó a través de gráficas de nube los resultados. Finalmente se, realizaron las recomendaciones y conclusiones a la luz de los objetivos trazados. Concluida esta fase, se procedió a la elaboración del informe final, así como del artículo producto del trabajo realizado (El Kadi, Pelekais, Robles y Leal, 2014, p. 79; Lechuga, 2018, p. 196, a).

\section{Resultados}

Las categorías analizadas fueron: Diagnóstico de la diversificación (acceso a mercados), diagnóstico de la diversificación (alcance geográfico), mecanismos de promoción comercial para la diversificación (acceso a mercados), mecanismos de promoción comercial para la diversificación (competitividad), mecanismos de promoción comercial para la diversificación (gobernabilidad), identificación de los factores de diversificación (factores financieros, humanos y sociales), evaluación de diversificación en empresas (diversificación), y comportamiento de factores de diversificación (diversificación). 


\subsection{Diagnóstico de la diversificación (acceso a mercados)}

\section{Tabla 2}

Importancia del conocimiento de los acuerdos comerciales

\begin{tabular}{lcc}
\hline Palabra & Frecuencia & $\begin{array}{c}\text { Frencuencia } \\
\text { Relativa }(\%)\end{array}$ \\
\hline Tratados & 7 & 1,44 \\
Países & 6 & 1,23 \\
Comercial & 5 & 1,03 \\
Exportar & 5 & 1,03 \\
Libre & 5 & 1,03 \\
Ventajas & 5 & 1,03 \\
\hline
\end{tabular}

Fuente: Elaboración propia (2019) a través de Nvivo 11.

Como parte de la revisión de datos, se procedió a identificar cuáles eran las palabras más frecuentes utilizando el programa Nvivo 11 sobre la importancia de conocer los acuerdos comerciales donde la palabra que asociaban con mayor periodicidad en las entrevistas es tratados, además de las palabras países, comercial, exportar, libre y ventajas (Ver tabla 2).

Resaltan la importancia de conocer los acuerdos comerciales con los que cuenta México al incrementar sus ventas en diferentes mercados, se expresa la falta de conocimiento de los empresarios y funcionarios de gobierno de los acuerdos comerciales, y dada la exención arancelaria en su uso permiten diversificar y tener acceso al mercado con precios competitivos a diferencia de los países con que no se cuenta acuerdo comercial, así como permite reducir los costos de transferencia entre países.

No obstante, Puerto (2010) asegura que uno de los retos que plantea la globalización económica para las empresas es, la creciente competencia en precios que exige a las empresas mayores niveles de productividad y eficiencia (p. 178).

\subsection{Diagnóstico de la diversificación (alcance geográfico)}

Tabla 3

Importancia de la diversificación de exportaciones

\begin{tabular}{ccc}
\hline Palabra & Frecuencia & $\begin{array}{c}\text { Frencuencia } \\
\text { Relativa (\%) }\end{array}$ \\
\hline México & 14 & 1,30 \\
Mercados & 13 & 1,21 \\
Países & 12 & 1,12 \\
Diversificación & 11 & 1,02 \\
Productos & 11 & 1,02 \\
\hline Fuente: Elaboración propia (2019). a través de Nvivo 11.
\end{tabular}

La percepción de los expertos mostró las siguientes palabras México, mercados, diversificación, países y productos. Resaltan la dependencia comercial de México a Estados Unidos por su cercanía geográfica y dinamismo económico, el modelo económico ha servido para el caso de México, pero la dependencia implica que ante fluctuaciones en la política comercial la economía mexicana puede verse afectada y por ende es necesaria la diversificación, de modo que al buscar la diversificación no se dependería de un único mercado (Ver tabla 3).

En este sentido sustenta la Organización de las Naciones Unidas para la Alimentación y la Agricultura - FAO (2004) en ausencia de diversificación de exportaciones; caídas y fluctuaciones de los ingresos de exportación han afectado negativamente al ingreso, la inversión y el empleo en los países en desarrollo. En contraste con la 
diversificación, los riesgos de la inversión se distribuyen entre más sectores económicos, lo que redunda a la larga en un incremento del ingreso (Acemoglu y Zilibotti, 1997).

\subsection{Mecanismos de promoción comercial para la diversificación (acceso a mercados)}

Tabla 4

\begin{tabular}{ccc}
\multicolumn{3}{c}{$\begin{array}{l}\text { Aprovechamiento de los acuerdos comerciales por } \\
\text { parte de las empresas exportadoras de Nuevo León }\end{array}$} \\
\hline Palabra & Frecuencia & $\begin{array}{c}\text { Frencuencia } \\
\text { Relativa (\%) }\end{array}$ \\
\hline Empresas & 18 & 2,31 \\
Acuerdos & 15 & 1,93 \\
México & 9 & 1,16 \\
Productos & 7 & 1 \\
\hline
\end{tabular}

Fuente: Elaboración propia (2019) a través de Nvivo 11.

La percepción de los expertos mostró las siguientes palabras empresas, acuerdos, México y productos. Expresan que las empresas grandes están aprovechando los acuerdos, sin embargo, las MiPymes por falta de conocimiento los están desaprovechando. Explican que un factor que incide son los costos logísticos de transporte al encontrarse la región a pocas horas de los Estados Unidos, los costos logísticos disminuyen siendo más atractivo este mercado que otros países como por ejemplo Centro y Suramérica. Por tanto, a pesar de que México cuenta con 12 acuerdos comerciales con 46 países, el 80\% de sus exportaciones son dirigidas a países Tratado de libre comercio México, Estados Unidos y Canadá - T-MEC. Otro fenómeno que explica la concentración es que la zona noreste tiene una estructura económica con vocación industrial y por tanto los inversionistas aprovechan la ventaja de costos de mano de obra barata para operar bajo el modelo de maquila y reimportarlo para su comercialización. Adicionalmente los acuerdos no sólo han disminuido el arancel a 0 sino que gozan de mecanismos de facilitación comercial a nivel aduanal como el derecho al trámite aduanal, no obstante, en la práctica se observa mal uso de los acuerdos cuando en algunas industrias entran productos a la zona T-MEC provenientes de China grabados con arancel, pero declarados en su origen como centroamericanos por colocar un ejemplo y luego se comercializan como nacionales (Ver tabla 4).

Como explican Rodil y López (2011) uno de los determinantes del comportamiento del sector exterior de México es su elevada concentración sectorial. la mayor parte de dichas exportaciones están asociados a la presencia de capital extranjero, fundamentalmente de origen estadounidense, siguiendo en este sentido un patrón de comportamiento ligado a las estrategias productivas propias de este capital transnacional (p.263). (López y Rodil, 2008).

Por otro lado, Alarcón (2007) explica que décadas después de la apertura comercial en México en la estructura productiva de Nuevo León, se ha registrado un cambio importante con la incorporación en gran escala de la industria maquiladora de exportación, de modo que esta ha adquirido características de industria globalizada, debido a que la producción de bienes o servicios de las empresas que la constituyen forma parte de cadenas productivas que se extienden generalmente por varios países, principalmente para la proveeduría en el mercado internacional (p. 65). 


\subsection{Mecanismos de promoción comercial para la diversificación (competitividad)}

Tabla 5

\begin{tabular}{ccc}
$\begin{array}{c}\text { Obstáculos aprovechamiento de acuerdos comerciales } \\
\text { para empresas exportadoras de Nuevo León }\end{array}$ \\
\hline Palabra & Frecuencia & $\begin{array}{c}\text { Frencuencia } \\
\text { Relativa (\%) }\end{array}$ \\
\hline Empresas & 9 & 1,02 \\
Mercado & 9 & 1,02 \\
México & 8 & 1 \\
\hline
\end{tabular}

Fuente: Elaboración propia (2019) a través de Nvivo 11.

La percepción de los expertos mostró las siguientes palabras empresas, mercado, y México. Como obstáculos destacan la falta de información, desconocimiento de los acuerdos por parte de los empresarios e incluso líderes gubernamentales, recurso humano calificado, apoyo financiero del gobierno, costos logísticos de transporte, falta infraestructura portuaria y navieras propias, manejo del inglés, conocimiento de los mercados extranjeros, barreras no arancelarias como simplificación de trámites y cumplimiento de normas internacionales (Ver tabla 5).

En efecto Francis y Collins-Dodd (2004) argumentan que muchas empresas son incapaces de realizar las exportaciones agresivamente por la carencia de experiencia, por sus recursos limitados o por otro obstáculo percibido: barreras para la exportación (Wheeler, 1990; Lechuga-Cardozo et al, 2014, p. 16).

\subsection{Mecanismos de promoción comercial para la diversificación (gobernabilidad)}

Tabla 6

Política Comercial de México y Mecanismos de Promoción Comercial en Nuevo León

\begin{tabular}{ccc}
\hline Palabra & Frecuencia & $\begin{array}{c}\text { Frencuencia } \\
\text { Relativa (\%) }\end{array}$ \\
\hline Comercio & 18 & 2,05 \\
México & 11 & 1,25 \\
Países & 9 & 1,02 \\
Promoción & 9 & 1,02 \\
\hline
\end{tabular}

Fuente: Elaboración propia (2019) a través de Nvivo 11.

La percepción de los expertos mostró las siguientes palabras comercio, México, países y promoción (Ver tabla 6). Se hace mención que la política comercial internacional de México es abierta un modelo neoliberal de libre competencia desde la firma del Acuerdo General sobre Aranceles Aduaneros y Comercio - GATT, México cuenta con 12 acuerdos comerciales, sin embargo, existe una descapitalización de los acuerdos, a excepción de T-MEC. Enfatizan a México como un socio comercial estratégico por su cercanía y posición en América del Norte.

Destacan el papel como organismos de promoción comercial en México Bancomext y Proméxico, el primero dedicado a la financiación de operaciones de comercio exterior y el segundo a la promoción de exportaciones. Así como existen organismos locales como Asociación de Empresarios Mexicanos en Estados Unidos - AEM, Consejo Mexicano Empresarial de Comercio Exterior COMCE Noreste, y Secretaría de Economía en su dirección de Inversión Extranjera y de Promoción de negocios. Con programas que facilitan e impulsan la comercialización de los productos en el mercado exterior.

Así como bien define Martínez (2007) las medidas de política pública que actual o potencialmente conducen a la actividad exportadora al nivel empresarial, industrial o nacional suministrados por el gobierno, asociaciones comerciales $u$ otras organizaciones (organismos públicos, privados o mixtos) (p.7) para ayudar a las empresas a 
superar barreras en la exportación, especialmente, a las Pymes (Calderón-García y Fayos-Gardó, 2002; Manera y Martín, 2006).

\subsection{Identificación de los factores de diversificación (factores financieros, humanos y sociales) Tabla 7}

Factores que inciden en la diversificación de exportaciones en Nuevo León

\begin{tabular}{ccc}
\hline Palabra & Frecuencia & $\begin{array}{c}\text { Frencuencia } \\
\text { Relativa (\%) }\end{array}$ \\
\hline Productos & 12 & 1,32 \\
Empresas & 9 & 1 \\
Mercado & 8 & 0,9 \\
\hline Fuente: Elaboración propia (2019) a través de Nvivo 11.
\end{tabular}

La percepción de los expertos mostró las siguientes palabras productos, empresas, y mercado (Ver tabla 7). Mencionan que los factores que inciden son la búsqueda de nuevos mercados, costos logísticos de transporte, posición geográfica, la calidad y precio de los productos, la innovación, dominio de un segundo idioma de los comerciales, conectividad portuaria, infraestructura vial, economías de escala, alianzas estratégicas, aranceles, la incertidumbre y existencia de un mercado potencial para los productos.

Cabe resaltar que son varios los elementos que pueden llevar a una empresa a internacionalizarse, como lo asegura Flor y Oltra (2010) "comportamiento de la empresa exportadora ha captado el interés de numerosas investigaciones, buena parte de las cuales se han destinado a identificar la influencia que diferentes factores ejercen sobre el resultado de su actividad exterior, al que denomina desempeño exportador" (p. 15). Así estudios como los de Escandón y Hurtado (2014) y Fernández, Peña y Hernández (2008) explican factores y variables que inciden en la orientación exportadora de la empresa. En efecto Huerta, Navas y Almodóvar (2004) quienes hacen una relación con la teoría de recursos y capacidades, calcifican en dos grandes grupos a los factores que inciden en la diversificación de exportaciones como Factores Externos al beneficio de una empresa es consecuencia tanto de las características competitivas del entorno y Factores Internos como de la combinación de los recursos de que dispone (p.89).

\subsection{Evaluación de diversificación en empresas (diversificación)}

Tabla 8

Evaluación e indicadores de la Diversificación de Exportaciones

\begin{tabular}{ccc}
\hline Palabra & Frecuencia & $\begin{array}{c}\text { Frencuencia } \\
\text { Relativa (\%) }\end{array}$ \\
\hline Mercado & 9 & 1 \\
Diversificación & 8 & 0,9 \\
México & 8 & 0,9 \\
Países & 8 & 0,9 \\
Productos & 8 & 0,9 \\
\hline
\end{tabular}

Fuente: Elaboración propia (2019) a través de Nvivo 11.

La percepción de los expertos mostró las siguientes palabras mercado, diversificación, México, países y productos (Ver tabla 8). Aseguran que comparada con otros países la diversificación es baja, en cuanto a la región comparada con otras regiones es alta, por la cercanía geográfica y cultural con Texas, para medirla se encuentra las estadísticas de la Secretaría de Economía. Existe falta de información y cumbres empresariales en cuanto al tema. Los indicadores que utilizarían serían el número de tratados, número de países, número de productos, 
número de sectores, volumen de importaciones y exportaciones, balanza comercial y remesas a partir de estos realizar un comparativo con economías desarrolladas.

Ahora bien, de acuerdo con información oficial de Comce Noreste "si se observa de un año a la fecha EE. UU. representaba el $80,5 \%$ de las exportaciones de Nuevo León, y dos años después representa el 78,5\% si bien son dos puntos porcentuales, hay cierto avance, y hay otros países que han crecido en un menor monto económico, pero si han crecido pues tenemos un caso como la India que ha aumentado al 100\%".

Así bien lo explica Mercier (2005) quien realiza un estudio de la industria maquiladora de exportación en México; y Mendoza (2006) quien realiza un estudio acerca de la integración económica de las ciudades de la frontera México Estados Unidos.

\subsection{Comportamiento de factores de diversificación (diversificación)}

Tabla 9

Similitudes y diferencias en el comportamiento de la diversificación de exportaciones de Nuevo León con otras regiones de América Latina

\begin{tabular}{ccc}
\hline Palabra & Frecuencia & $\begin{array}{c}\text { Frencuencia } \\
\text { Relativa (\%) }\end{array}$ \\
\hline Países & 15 & 1,54 \\
México & 14 & 1,44 \\
Argentina & 10 & 1,03 \\
Productos & 10 & 1,03 \\
Brasil & 9 & 1 \\
Valor & 9 & 1 \\
\hline
\end{tabular}

Fuente: Elaboración propia (2019) a través de Nvivo 11.

La percepción de los expertos mostró las siguientes palabras países, México, Argentina, productos, Brasil, y valor (Ver tabla 9). Se hace mención que las similitudes es en cuanto a la canasta de productos básicos que se exportan y la falta de desarrollo tecnológico. En cuanto a las diferencias México está desarrollando su industria automotriz, aeronáutica y la industria del conocimiento la mano de obra está migrando a mano de obra calificada. Agregan los socios comerciales de los países de américa latina guardan cercanía geográfica. México se encuentra por encima del promedio de la diversificación de exportaciones en América Latina.

Lo anterior se sustenta según Pérez-Escatel y Pérez-Veyna (2009) en que, a partir de la apertura comercial, con el ingreso al Acuerdo General sobre Aranceles Aduaneros y Comercio en 1986, México ha incrementado sus volúmenes de exportación, con ello se han incrementado el número de empresas exportadoras y, consecuentemente, éstas han mejorado la calidad de sus productos y hecho eficiente su producción, debido a un aumento en las capacidades tecnológicas. Por tanto, este desempeño tecnológico se explica como resultado de la apertura comercial (p.159).

Agregan Contreras y Munguía (2007) la expansión de las maquiladoras ha generado una parte sustancial del crecimiento industrial en el norte de México, durante las últimas tres décadas, que inicialmente se constituyeron en una estrategia temporal del gobierno mexicano para hacer frente al desempleo en la zona fronteriza con EE. UU., no obstante, hoy por hoy se constituyen en pieza fundamental del esquema económico soportado en la política comercial del país. Comentan Carrillo (2004); Contreras y Hualde (2004) en la actualidad muchas ya cuentan con tecnologías de punta, organización moderna y fuerza de trabajo calificada que incluye técnicos e ingenieros. 


\section{Conclusiones}

En los apartados anteriores se ha podido comprender cuál es el estado actual del uso de los acuerdos comerciales en Nuevo León (México) y, a partir de las opiniones de los expertos, cuál es la situación en lo que respecta a la diversificación de exportaciones y los mecanismos de promoción utilizados en esta región. Por lo que se aporta la siguiente explicación gráfica del fenómeno (ver ilustración 1).

\section{Ilustración 1}

Modelo de Relaciones Actores y Variables de Promoción Comercial en Nuevo León

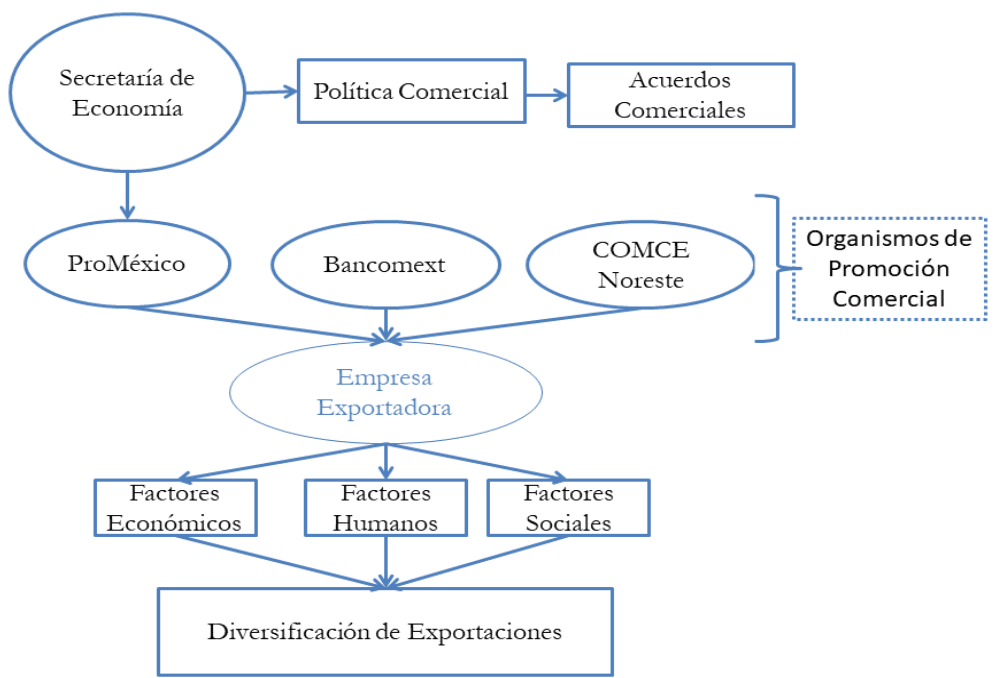

Fuente: Elaboración propia, (2019).

La Secretaría de Economía es el ente federal encargado de administrar la política comercial internacional, que en el marco de su apertura comercial cuenta con 12 acuerdos comerciales, estos acuerdos son promocionados por ProMéxico entidad encargada de la promoción de las exportaciones y la inversión extrajera en México en conjunto con Bancomext encargado de la financiación para la participación de las empresas exportadoras en las ferias comerciales internacionales y financiamiento de las operaciones de comercio exterior mexicanas, en Nuevo León se encuentra Comce Noreste iniciativa privada para la promoción de exportaciones e inversión en el estado. Las empresas exportadoras que reciben el apoyo de las entidades mencionadas dependen de los factores económicos, humanos y sociales que inciden en que diversifiquen o no sus exportaciones hacia los mercados exteriores.

Por lo que se refiere a la economía mexicana a partir de la década de los ochenta ha practicado una política de regionalismo abierto, con la que ha ganado paulatinamente apertura comercial, lo que ha permitido alcanzar una red de acuerdos comerciales con distintos países, con los cuales mayoritariamente ha desarrollado su comercio. Sustenta Bouszas (2017) buena parte del comercio de América Latina se encuentra concentrado en México, y fue de las primeras economías en extender la generación de acuerdos comerciales a través del TLCAN.

En definitiva, sobre la base de los resultados obtenidos con la realización de las entrevistas y la revisión documental, se pueden enumerar algunas conclusiones generales:

1. Diagnóstico de la diversificación. Los expertos coinciden en que existe desconocimiento de los empresarios y funcionarios de gobierno para el aprovechamiento de los acuerdos comerciales, su uso permite el 
aprovechamiento de preferencias arancelarias, acceso a mercados a precios competitivos, y reducción de costos de transferencia a precios competitivos. Agregan que para el caso de México existe una dependencia comercial con su vecino Estados Unidos, la concentración de exportaciones hacia este país como se observa en la tabla 1, ello implica que ante fluctuaciones en los flujos de cambio la economía mexicana puede verse afectada por su dependencia directa, por ende, es de vital importancia moverse hacia un escenario diversificado (Puerto, 2010; la Organización de las Naciones Unidas para la Alimentación y la Agricultura - FAO, 2004; Acemoglu y Zilibotti, 1997).

2. Mecanismos de promoción comercial. Los expertos explican que un factor que incide en el desaprovechamiento de la totalidad de los acuerdos comerciales son la ventaja en costos logísticos de transporte derivados de la proximidad geográfica con Texas, aunado a la estructura industrial basada en el modelo de maquila que facilita el aprovechamiento de la ventaja de costos de mano de obra por parte de inversionistas de Estados Unidos y Corea del Sur principalmente.

Como obstaculizadores del aprovechamiento de acuerdos comerciales por parte del sector empresarial destacan los expertos: Desconocimiento de los acuerdos comerciales, recurso humano calificado, apoyo financiero gubernamental, costos logísticos de transporte, ausencia de infraestructura portuaria, manejo del inglés, conocimiento de los mercados extranjeros, y barreras no arancelarias.

Por otro lado, los organismos de promoción públicos en México son Bancomext, Proméxico y Secretaría de Economía. Los privados son Consejo Mexicano Empresarial de Comercio Exterior COMCE y Asociación de Empresarios Mexicanos en Estados Unidos - AEM, tanto públicos como privados tienen por función diseñar y ejecutar programas encaminados a la promoción de exportaciones, financiación de operaciones de comercio exterior, atracción de la inversión extrajera directa e impulso de la comercialización de los productos y servicios nacionales en el mercado exterior(Rodil y López, 2011; López y Rodil, 2008; Alarcón, 2007; Francis y Collins-Dodd, 2004; Wheeler, 1990; Lechuga-Cardozo et al, 2014; Martínez, 2007; Calderón-García y Fayos-Gardó, 2002; Manera y Martín, 2006).

3. Identificación de los factores de diversificación. Los expertos mencionan que los factores que inciden en la diversificación de exportaciones son entre otros la búsqueda de nuevos mercados, costos logísticos de transporte, posición geográfica, la calidad y precio de los productos, la innovación, el dominio de un segundo idioma, conectividad portuaria, infraestructura vial, economías de escala, alianzas estratégicas, aranceles, la incertidumbre y existencia de un mercado potencial para los productos(Flor y Oltra, 2010; Escandón y Hurtado (2014); Fernández, Peña y Hernández, 2008; Huerta, Navas y Almodóvar, 2004).

4. Evaluación de diversificación en empresas. Los expertos consideran que indicadores para medir la diversificación de exportaciones son el número de tratados, número de países, número de productos, número de sectores, volumen de importaciones y exportaciones, balanza comercial y remesas. La diversificación de exportaciones en la región se considera baja si se tiene en cuenta que el 78,2\% de las exportaciones tienen como destino América del Norte, aprovechándose en mayor medida el T-MEC. Sin embargo, existe un interés latente de las empresas en Nuevo León por ir conquistando poco a poco el mercado asiático y europeo, impulsado por el cambio en las políticas comerciales

Promovidas por el actual gobierno de EE. UU. que se hicieron evidentes en la renegociación del TLCAN, oportunidad de reflexión para las empresas del riesgo que implica depender nada más de un solo mercado o de un cliente (Mercier, 2005; Mendoza, 2006).

5. Comportamiento de factores de diversificación. En cuanto a similitudes con otras economías latinoamericanas existe coincidencia en canasta exportable de productos y la ausencia de desarrollo tecnológico intensivo en ellos. En contraste en contraste en México se encuentran grandes avances en la industria automotriz y aeronáutica, y 
la mano de obra está migrando hacia mano de obra calificada (Pérez-Escatel y Pérez-Veyna, 2009; Contreras y Munguía, 2007; Carrillo, 2004; Contreras y Hualde, 2004).

A partir de las reflexiones de los expertos para el aprovechamiento efectivo de la totalidad de los acuerdos comerciales en México se sugiere al sector empresarial: 1. Explorar oportunidades comerciales para productos específicos en mercados que cuenten con trato arancelario preferencial (Maldonado, 2014); 2. Aprovechar las capacidades de las empresas grandes para el apalancamiento de Pymes en su inserción exitosa en los mercados internacionales a través de figuras como clúster (Valencia, 2015; Garzón, 2015), y 3. Ocupar segmentos de mercado de forma asociativa, o entregar productos a redes exportadoras por la capacidad financiera y tecnológica para penetrar mercados complejos y exigentes de economías desarrolladas(Lozano, 2010; Lechuga y Leyva, 2019).

Finalmente, podemos afirmar que la presente investigación sirve como paso previo para futuras líneas de investigación, que incluyan una investigación empírica encaminada a profundizar en el conocimiento del aprovechamiento de los acuerdos comerciales en la región. Siendo cociente de ello se considera oportuno dar continuidad a este estudio planteando como futuras líneas de trabajo el desarrollo de una investigación empírica sobre los acuerdos comerciales que incluya los actores como el Estado, las empresas y los organismos de promoción comercial que pueda facilitar datos cuantitativos y estadísticamente relevantes.

\section{Referencias bibliográficas}

Acemoglu, D., y F. Zilibotti (1997). Was Prometheus unbound by chance? Risk diversification and growth. Journal of Political Economy, 105(4), 709-751.

Agosin, M. (2009). Crecimiento y diversificación de exportaciones en economías emergentes. Revista de la Cepal, 97, $117-134$.

Acosta, J. y Arango, J. (1994). La relación entre crecimiento y diversificación de las exportaciones y desarrollo económico: reflexiones y recomendaciones para incrementar las exportaciones. Colombia Internacional, 26, 1- 11.

Alarcón Martínez, G. (2007). Oportunidad y Desafío. Nuevo León en el umbral de su cuarta industrialización. Trayectorias, 9(25), 61-77.

Álvarez-López, M. E., y Myro, R. (2018). Diversificación de mercados y crecimiento de la exportación. Papeles de Economía Española, (158), 90 - 101.

Aulakh, P. S.; Kotabe, M. y Teegen, H. (2000). Export Strategies and Performance of Firms from Emerging Economies: Evidence from Brazil, Chile And Mexico. Academy of Management Journal, 43 (3), 342-361.

Benito, G. R. G, y Welch, L. S. (1994). Foreign market servicing: beyond choice of entry mode. En Journal of International Marketing, 2, 7-28.

Bernal, C. (2010). Metodología de la investigación. Bogotá: Pearson.

Bouzas, R. (2017). El regionalismo en América Latina y el Caribe: ¿qué hay de nuevo? Estudios Internacionales, $49,65-88$.

Cabrera, M. y Olivares, A. (2012). La influencia de los recursos de capital humano, social y financiero sobre la velocidad del proceso exportador de las empresas familiares. En Revista Europea de Dirección y Economía de la Empresa, 21, 306-315. 
Calderón-García, H. y Fayos-Gardó, T. (2002). La medición de los resultados de la promoción de las exportaciones. Boletín ICE Económico: Información Comercial Española, 2746, 35-42.

Carlsen, B. y Glenton, C. (2011). What about N? A methodological study of sample-size reporting in focus group studies. BMC Medical Research Methodology ,11(26), 2-10.

Carrillo, J. (2004.). Principales estadísticas de la industria maquiladora. Encuesta sobre aprendizaje tecnológico y escalamiento industrial. Tijuana (México): El Colegio de la Frontera Norte.

Casas, J. (2015). Diversificación de las exportaciones y su impacto sobre el crecimiento económico en Colombia (Tesis de pregrado). Universidad del Valle, Cali (Colombia).

Contreras, Ó. y Hualde, A. (2004). El aprendizaje y sus agentes. Los portadores del conocimiento en las maquiladoras del norte de México. Estudios Sociológicos, 22 (64), 79-121.

Contreras, O. y Munguía, L. (2007). Evolución de las maquiladoras en México. Política industrial y aprendizaje tecnológico. Región y sociedad, 19, 71 -87.

Cooper, R. G. y Kleinschmidt, E. J. (1985). The impact of export strategy on export sales performance. Journal of International Business Studies, Spring, 37-55.

Córdoba, M. (2015). Finanzas Internacionales. Bogotá: Ecoe Ediciones.

Creswell, J. W., \& Miller, D. L. (2000). Determining validity in qualitative inquiry. Theory into Practice, 39(3), 124-130.

Davis, P. y Harveston, P. (2000). Internationalization and organizational growth: The impact of internet usage and technology involvement among entrepreneur-led family businesses. Family Business Review, 13, 107120.

Deardoff, A. (1984). Testing trade theories and predicting trade flows. En R.W. Jones \& P.B. Kenen (Eds.), Handbook of International Economics. Amsterdam: Elsevier Science Publishers, B.V.

El Kadi, O., Pelekais, C., Robles, M. y Leal, M. (2015). Autotomía caudal como estrategia gerencial para el cambio organizacional en empresas familiares. Desarrollo Gerencial, 7(1). 68-84.

Escandón, D. y Hurtado, A. (2014). Factores que influyen en el desarrollo exportador de las pymes en Colombia. En Estudios Gerenciales, 30, 172-183.

Fernández, M.; Peña, I. y Hernández, F. (2008). Factores determinantes del éxito exportador. El papel de la estrategia exportadora en las cooperativas agrarias. En Revista de Economía Pública, Social y Cooperativa, 63, 39-64.

Flor, M. y Oltra, M. (2010). La estrategia exportadora de la empresa y su relación con el resultado internacional. En Investigaciones Europeas de Dirección y Economía de la Empresa, 16 (1), 15-29.

Francis, J. y Collins-Dodd, C. (2004). Impact of export promotion programs on firm competencies, strategies and performance. The case of Canadian high technology SMEs. International Marketing Review, 21 (4/5), 474495.

García-Hernández, G.E. y Manzano-Caudillo, J. (2010). Procedimientos metodológicos básicos y habilidades del investigador en el contexto de la teoría fundamentada. Iztapalapa. Revista de ciencias sociales y humanidades, 69, 17-39. 
Garzón Castrillón, M. (2015). Modelo de Capacidades Dinámicas. Dimensión Empresarial, 13 (1), 11 -131.

Giraldo-Isaza, F. (2006). Antecedentes y posibles ventajas y desventajas del Tratado de Libre Comercio - TLC para un país como Colombia. Apuntes del CENES, 25 (42), 1 -27.

Gómez, A. (2013). ¿Cuál es el pasado y presente de la apertura comercial de México? Recuperado de: http://www.dineroenimagen.com/2013-05-27/20763

Guardia, A. (2009). Lineamientos de una estrategia exportadora para el aprovechamiento de los acuerdos comerciales y las cadenas productivas. En M. Jelvez (Ed.), Perspectivas Económicas para el Chile del Bicentenario: Desafíos y Oportunidades (pp. 55-80). Santiago de Chile: Cepal.

Hernández, R., Fernández, C. y Baptista, P. (2014). Metodología de la investigación. México: Mc Graw Hill.

Huerta-Riveros, P.; Navas-López, J. y Almodóvar- Martínez, P. (2004). La Diversificación desde la Teoría de Recursos y Capacidades. Cuadernos de Estudios Empresariales, 14, 87-104.

Hurtado, J. (2010). Guía para la comprensión holística de la ciencia. Caracas (Venezuela): Fundación Sypal, p. 133.

Lamb, W.; Hair, J. y McDaniel, C. (2006). Marketing Edición Latinoamérica. Ciudad de México: Cenage Learning.

Lechuga- Cardozo, J. (2016). Módulo de Finanzas Internacionales. Soledad (Colombia): Instituto Tecnológico de Soledad Atlántico.

Lechuga-Cardozo, J. (2018, a). Necesidades insatisfechas de una comunidad aledaña a una terminal portuaria de Barranquilla. Revista Pensamiento y Gestión, 45 (2) 181-210.

Lechuga-Cardozo, J. (2018, b). Industria de la madera en Colombia: Recursos claves para el resultado exportador (Wood Industry in Colombia: Key Resources for Export Performance). Revista Academia \& Negocios, 4(1), $15-24$.

Lechuga-Cardozo, J., y Leyva-Cordero, O. (2019). Escenarios 2020 del Orden Mundial. Análisis desde la Prospectiva Estratégica. ÁNFORA, 27(48), 137-161. https://doi.org/10.30854/anf.v27.n48.2020.672

Lechuga, J., Rodríguez, M. y Alarcón, C. (2014). Estado de conocimiento que tienen las Instituciones de Educación Superior (IES) tecnológicas de Barranquilla y Cartagena sobre sus grupos de interés. Revista de Investigaciones UNAD, 13(2), 171-187.

Lombana Coy, J.; Rozas Gutiérrez, S.; Corredor Velandia, C.; Silva Guerra, H.; Castellano Ramírez, A. Gonzales Ortiz, J. y Ortiz Velásquez, M. (2013). Negocios internacionales: fundamentos y estrategias. Barranquilla: Ecoe Ediciones-Universidad del Norte.

López-Cózar, C.; Priede, T. y Benito, S. (2013). Influencia de la deuda en la estrategia de exportación de la empresa familiar. En Revista de Empresa Familiar, 3(1), 31-46.

López, D.; López, E. y Montes, E. (2015). Colombia en el comercio mundial (1992-2012): desempeño de las exportaciones colombianas. Borradores de Economía, 885. Bogotá (Colombia): Banco de la República.

López, J. y Rodil, O. (2008). El comercio intraindustrial e intra-firma en México durante el proceso de integración de América del Norte (1993-2006). Economía UNAM, 5(13), 86-112. 
Lozano Monroy, F. (2010). La asociatividad como modelo de gestión para promover las exportaciones en las pequeñas y medianas empresas en Colombia. Revista de Relaciones Internacionales, Estrategia y Seguridad, 5 (2), $161-191$.

Maldonado-Narváez, M. (2014). Las reglas de origen en los tratados de libre comercio.

Manera-Bassa, J. y Martín del Peso, M. (2006). Percepción de los instrumentos de promoción comercial de apoyo a la internacionalización de las empresas en las comunidades autónomas de España. Tribuna Económica, 828, 249 - 268.

Martín, J. (2004). Formas de entrada en los mercados exteriores. Boletín Económico del ICE, Información Comercial Española, 2823, 33-48.

Martínez, P. (2007). Influencia de la promoción de exportaciones en el proceso del desarrollo exportador de las Pymes. Un estudio de caso. Pensamiento \& Gestión, 23, 1-57.

Martínez-Salgado, C. (2012). El muestreo en investigación cualitativa. Principios básicos y algunas controversias. Ciência \& Saúde Coletiva, 17(3), 613-619.

Mendoza-Cota, J. (2006). La integración económica de las ciudades de la frontera México- Estados Unidos. Análisis Económico, 21 (46), 307-325.

Mendoza, R. (2006). Investigación cualitativa y cuantitativa. Diferencias y limitaciones. Recuperado de http://www.gycperu.com

Mercier, D. (2005). La industria maquiladora de exportación mexicana hace 40 años. Revista Galega de Economía, 14 (2), 1-17.

Organización de las Naciones Unidas para la Agricultura y la Alimentación - FAO (2004). El estado de los mercados de productos básicos agrícolas 2004. Roma: Autor.

Pérez-Escatel, A. y Pérez-Veyna, O. (2009). Competitividad y acumulación de capacidades tecnológicas en la industria manufacturera mexicana. Investigación económica, 68, 159-187.

Piercy, N. (1982). Export strategy: Markets and competition. Londres: Allen y Unwin.

Puerto-Becerra, D. (2010). La globalización y el crecimiento empresarial a través de estrategias de internacionalización. Pensamiento \& Gestión, (28), 171-195.

Rodil-Marzábal, Ó., y López-Arévalo, J. A. (2011). Efectos del Tratado de Libre Comercio de América del Norte sobre el comercio de México: creación de comercio y especialización intraindustrial. Revista de economía mundial, 27, 249-278.

Rodríguez, K. y Gorjón, J. (2014). Grupos de Enfoque. En K. Saénz-López \& G. Tamez-González (Eds.), Métodos y técnicas cualitativas y cuantitativas aplicables a la investigación en ciencias sociales. México D.F.: Tirant Humanidades.

Sandelowski, M. \& Barroso, J. (2003). Writing the proposal for a qualitative research methodology project Qualitative. Health Research, 13(6), 781-820.

Schiavon, J. y Ortiz, A. (2001). Apertura comercial y reforma institucional en México (1988-2000): un análisis comparado del TLCAN y el TLCUE. Foro Internacional, 41 (4), 731-760. 
Secretaría de Economía México (2015). Comercio Exterior / Países con Tratados y Acuerdos firmados con México. https://www.gob.mx/se/acciones-y-programas/comercio-exterior-paises-con-tratados-yacuerdos-firmados-con-mexico

Secretaría de Economía y Trabajo del Estado de Nuevo León (2018). Exportaciones Manufactureras Mensuales. http://datos.nl.gob.mx/n-I-exportaciones-manufactureras-mensuales/

Seringhaus, F.H.R. (1986). The impact of government export marketing assistance. International Marketing Review, 3 (2), 55-66.

Solorza-Luna, M. (2012). Consecuencias económicas de los tratados comerciales entre América Latina y Estados Unidos. Ola Financiera, 6(14), 19 -36.

Strauss, A. y Corbin, J. (1990). Basics of qualitative research. P edición. Thousand Oaks: Sage.

Taylor, S.J. y Bogdan, R. (1986). Introducción: ir hacia la gente, en Introducción a los métodos cualitativos de investigación. México: Paidós, p. 20.

Trujillo-Acosta, I. (2014). Sobre las ventajas de la Alianza del Pacífico para Colombia. Revista Prolegómenos, Derechos y Valores, 17 (33), 159-172.

Valencia-Rodríguez, M. (2015). Capacidades dinámicas, innovación de producto e aprendizaje organizacional en pymes del sector cárnico. Ingeniería Industrial, 36 (3), 297 - 305.

Villareal, R. y Ramos, R. (2001). La apertura de México y la paradoja de la competitividad: hacia un modelo de competitividad sistémica. Comercio Exterior, 51(9), 772-788.

Vinesh, R.; Seetanah, B. y John, M. (2014). Diversificación de las exportaciones y crecimiento económico: el caso de Mauricio. Ginebra: Organización Mundial del Comercio.

Wheeler, C.N. (1990). Stimulating Scottish and United Kingdom economies through export promotion programs. En S.T. Cavusgil y M.R. Czinkota (Eds.), International Perspectives on Trade Promotion and Assistance (pp. 101-18), New York, NY: Quorum.

Esta obra está bajo una Licencia Creative Commons Attribución-NoCommercial 4.0 International

\section{(cc) BY-NC}

